\title{
Generalized measurements via programmable quantum processor
}

\author{
Marián Roško ${ }^{1}$, Vladimír Bužek ${ }^{1,2}$, Paul Robert Chouha ${ }^{3}$, and Mark Hillery ${ }^{3}$ \\ ${ }^{1}$ Research Center for Quantum Information, Slovak Academy of Sciences, 84511 Bratislava, Slovakia \\ ${ }^{2}$ Department of Mathematical Physics, National University of Ireland, Maynooth, Co. Kildare, Ireland \\ ${ }^{3}$ Department of Physics and Astronomy, Hunter College of CUNY, 695, Park Avenue, New York, NY 10021, U.S.A.
}

(September 17, 2003)

\begin{abstract}
We show that it is possible to control the trade-off between information gain and disturbance in generalized measurements of qudits by utilizing the programmable quantum processor. This universal quantum machine allows us to perform a generalized measurement on the initial state of the input qudit to construct a Husimi function of this state. The trade-off between the gain and the disturbance of the qudit is controlled by the initial state of ancillary system that acts as a program register for the quantum-information distributor. The trade-off fidelity does not depend on the initial state of the qudit.
\end{abstract}

\section{INTRODUCTION}

Recently in several experiments [1-3] optimal quantum cloning of qubits [4-6] has been achieved. In these experiments the information that was originally encoded in an (unknown) state $|\Psi\rangle$ of an input qubit has been distributed between two qubits in a covariant way (i.e., the fidelity of this information distribution does not depend on the state of the input qubit). Quantum cloning, viewed as a process of information distribution, can be considered as one of the basic tasks of quantuminformation processing (QIP). Another important task of QIP is the application of specific operations (maps) to the input data. In order to perform either of these tasks, we have to control the dynamics of the data register. This control can be achieved by having external forces, which are specified by classical parameters (e.g. phases and amplitudes of lasers), act on the quantum system [7-9]. Alternatively, the control of the dynamics of the data register can be performed on the quantum level, that is the maps induced on the data register can be completely specified by the quantum state of a program register in a quantum processor. The action of the processor is specified by a unitary operator acting on the Hilbert space of the data and the program register and results in a map induced on the data [10-14].

In this paper we will consider a specific model of the quantum processor - the so-called quantum-information distributor (QID), which was introduced recently in Ref. [15]. This covariant quantum processor allows us to distribute quantum information into several quantum channels as well as to perform specific quantum operations in each of the channels. This set up is interesting per se since it allows us to achieve quantum control over quantum systems. In addition, if the quantuminformation distributor is combined with a projective measurement performed on some of the output channels one can achieve interesting generalized quantum (positive operator value measure) measurements on the input register. In particular, in this paper we will show how quantum filtering of the original (input) data register can be realized and how propensities (e.g., a Husimi function) of the input register can be easily measured.

Our paper is organized as follows. In Sec. II we will introduce a formal description of a qudit and some basic operations that can be performed on a single qudit and controlled rotations that can be performed on two qudits. In Sec. III we will describe the quantuminformation distributor and the role of the programs encoded in states of program qudits. Sec. IV we will be devoted to a description of generalized measurements and the reconstruction (measurement) of the Husimi function in a discrete phase space. In Sec. V we will analyze how positive-operator value measure (POVM) measurement can be realized with the help of quantum information distributor. We conclude our paper with some remarks on the noise induced on the input data qudit due to the projective measurements performed on the program qudits at the output of quantum-information distributor.

\section{OPERATIONS ON QUDITS}

In order to make our discussion self-contained we first present a brief review of the formalism describing quantum states in a finite-dimensional Hilbert space. Here we follow the notation introduced in Ref. [16]. Let the $N$-dimensional Hilbert space be spanned by $N$ orthogonal normalized vectors $\left|x_{k}\right\rangle$ or, equivalently, by $N$ vectors $\left|p_{l}\right\rangle, k, l=0, \ldots, N-1$, where these bases are related by the discrete Fourier transform

$$
\begin{aligned}
& \left|x_{k}\right\rangle=\frac{1}{\sqrt{N}} \sum_{l=0}^{N-1} \exp \left(-i \frac{2 \pi}{N} k l\right)\left|p_{l}\right\rangle \\
& \left|p_{l}\right\rangle=\frac{1}{\sqrt{N}} \sum_{k=0}^{N-1} \exp \left(i \frac{2 \pi}{N} k l\right)\left|x_{k}\right\rangle .
\end{aligned}
$$

Without loss of generality, it can be assumed that these bases consist of sets of eigenvectors of non-commuting 
operators $\hat{X}$ and $\hat{P}$ :

$$
\hat{X}\left|x_{k}\right\rangle=k\left|x_{k}\right\rangle, \quad \hat{P}\left|p_{l}\right\rangle=l\left|p_{l}\right\rangle,
$$

that is,

$$
\hat{X}=\sum_{k=0}^{N-1} k\left|x_{k}\right\rangle\left\langle x_{k}\left|; \quad \hat{P}=\sum_{l=0}^{N-1} l\right| p_{l}\right\rangle\left\langle p_{l}\right| .
$$

For instance, we can assume that the operators $\hat{X}$ and $\hat{P}$ are related to a discrete "position" and "momentum" of a particle on a ring with a finite number of equidistant sites [17]. Specifically, we can introduce a length scale, $L$, and two operators, the position $\hat{x}$ and the momentum $\hat{p}$, such that

$$
\hat{x}\left|x_{k}\right\rangle=x_{k}\left|x_{k}\right\rangle, \quad \hat{p}\left|p_{l}\right\rangle=p_{l}\left|p_{l}\right\rangle,
$$

where

$$
x_{k}=L \sqrt{\frac{2 \pi}{N}} k ; \quad p_{l}=\frac{1}{L} \sqrt{\frac{2 \pi}{N}} l,
$$

where we have used units such that $\hbar=1$. The length, $L$ can, for example, be taken equal to $\sqrt{1 / \omega m}$, where $m$ is the mass and $\omega$ is the frequency of a quantum "harmonic" oscillator within a finite dimensional Fock space.

The squared absolute values of the scalar product of eigenkets (2.2) do not depend on the indices $k, l$ :

$$
\left|\left\langle x_{k} \mid p_{l}\right\rangle\right|^{2}=1 / N
$$

which means that pairs $(k, l)$ form a discrete phase space (i.e., pairs $(k, l)$ represent "points" of the discrete phase space) on which (quasi) probability density distributions associated with a given quantum state can be defined [18-22]. Next we introduce operators which shift (cyclicly permute) the basis vectors [23]:

$$
\begin{aligned}
& \hat{R}_{x}(n)\left|x_{k}\right\rangle=\left|x_{(k+n) \bmod N}\right\rangle ; \\
& \hat{R}_{p}(m)\left|p_{l}\right\rangle=\left|p_{(l+m) \bmod N}\right\rangle,
\end{aligned}
$$

where the sums of indices are taken modulo $N$ (this summation rule is considered throughout this paper, where it is clear we will not explicitly write the symbol $\bmod N$ ). For more about the properties of these operators and the role they play in the discrete phase space $(k, l)$ see Ref. [24].

A general single-particle state in the $x$ basis can be expressed as

$$
|\Psi\rangle_{1}=\sum_{k=0}^{N-1} c_{k}\left|x_{k}\right\rangle_{1} ; \quad \sum_{k=0}^{N-1}\left|c_{k}\right|^{2}=1 .
$$

The basis of maximally entangled two-particle states (the analog of the Bell basis for spin- $\frac{1}{2}$ particles) can be written as

$$
\left|\Xi_{m n}\right\rangle=\frac{1}{\sqrt{N}} \sum_{k=0}^{N-1} \exp \left(i \frac{2 \pi}{N} m k\right)\left|x_{k}\right\rangle\left|x_{(k-n) \bmod N}\right\rangle,
$$

where $m, n=0, \ldots, N-1$. We can also rewrite these maximally entangled states in the $p$ basis:

$$
\left|\Xi_{m n}\right\rangle=\frac{1}{\sqrt{N}} \sum_{l=0}^{N-1} \exp \left(-i \frac{2 \pi}{N} n l\right)\left|p_{(m-l) \bmod N}\right\rangle\left|p_{l}\right\rangle \text {. }
$$

The states $\left|\Xi_{m n}\right\rangle$ form an orthonormal basis

$$
\left\langle\Xi_{k l} \mid \Xi_{m n}\right\rangle=\delta_{k, m} \delta_{l, n},
$$

with

$$
\sum_{m, n=0}^{N-1}\left|\Xi_{m n}\right\rangle\left\langle\Xi_{m n}\right|=\hat{\mathbb{1}} \otimes \hat{\mathbb{1}} .
$$

In order to prove the above relations we have used the standard relation $\sum_{n=0}^{N-1} \exp \left[2 \pi i\left(k-k^{\prime}\right) n / N\right]=N \delta_{k, k^{\prime}}$.

It is interesting to note that the whole set of $N^{2}$ maximally entangled states $\left|\Xi_{m n}\right\rangle$ can be generated from the state $\left|\Xi_{00}\right\rangle_{23}$ by the action of local unitary operations (shifts), e.g.,

$$
\left|\Xi_{m n}\right\rangle_{23}=\hat{\mathbb{1}}_{2} \otimes \hat{R}_{x}^{\dagger}(n) \hat{R}_{p}(m)\left|\Xi_{00}\right\rangle_{23},
$$

acting just on system 3 in this particular case.

From the definition of the states $\left|\Xi_{m n}\right\rangle_{23}$ it follows that they are simultaneously eigenstates of the operators $\hat{X}_{2}-\hat{X}_{3}$ and $\hat{P}_{2}+\hat{P}_{3}$ :

$$
\begin{aligned}
\left(\hat{X}_{2}-\hat{X}_{3}\right)\left|\Xi_{m n}\right\rangle_{23} & =n\left|\Xi_{m n}\right\rangle_{23} \\
\left(\hat{P}_{2}+\hat{P}_{3}\right)\left|\Xi_{m n}\right\rangle_{23} & =m\left|\Xi_{m n}\right\rangle_{23} .
\end{aligned}
$$

We easily see that for $N=2$ the above formalism reduces to the well-known spin- $\frac{1}{2}$ particle (qubit) case.

Now we introduce generalizations of the two-qubit controlled-NOT (CNOT) gate (see also Ref. [25]). In the case of qubits the CNOT gate is represented by a two-particle operator such that if the first (control) particle labeled $a$ is in the state $|0\rangle$ nothing "happens" to the state of the second (target) particle labeled $b$. If, however, the control particle is in the state $|1\rangle$ then the state of the target is "flipped", i.e., the state $|0\rangle$ is changed into the state $|1\rangle$ and vice versa. Formally we can express the action of this CNOT gate as a two-qubit operator of the form

$$
\hat{D}_{a b}=\sum_{k, m=0}^{1}|k\rangle_{a}\langle k|\otimes|(m+k) \bmod 2\rangle_{b}\langle m| .
$$

We note that in principle one can introduce an operator $\hat{D}_{a b}^{\dagger}$ defined as

$$
\hat{D}_{a b}^{\dagger}=\sum_{k, m=0}^{1}|k\rangle_{a}\langle k|\otimes|(m-k) \bmod 2\rangle_{b}\langle m| .
$$


In the case of qubits these two operators are equal. This is not the case when the dimension of the Hilbert space is larger than two [25]. Let us generalize the above definition of the operator $\hat{D}$ for $N>2$. Before doing so, we shall simplify our notation. Because we will work mostly in the $x$ basis we shall use the notation $\left|x_{k}\right\rangle \equiv|k\rangle$, where it may be done so unambiguously. With this in mind we now write

$$
\hat{D}_{a b}=\sum_{k, m=0}^{N-1}|k\rangle_{a}\langle k|\otimes|(m+k) \bmod N\rangle_{b}\langle m| .
$$

From definition (2.17) it follows that the operator $\hat{D}_{a b}$ acts on the basis vectors as

$$
\hat{D}_{a b}|k\rangle|m\rangle=|k\rangle|(k+m) \bmod N\rangle,
$$

which means that this operator is equal to the conditional adder $[26,27]$ and can be performed with the help of a simple quantum network as discussed in Ref. [26].

If we take into account the definition of the shift operator $\hat{R}_{x}(n)$ given by Eq. (2.7) and the definition of the position and momentum operators $\hat{x}$ and $\hat{p}$ given by Eq. (2.4) we can rewrite the operator $\hat{D}_{a b}$ as:

$$
\begin{aligned}
\hat{D}_{a b} & =\sum_{k, m=0}^{N-1}|k\rangle_{a}\left\langle k\left|\otimes \hat{R}_{x}^{(b)}(k)\right| m\right\rangle_{b}\langle m| \\
& \equiv \sum_{k=0}^{N-1}|k\rangle_{a}\langle k| \otimes \hat{R}_{x}^{(b)}(k),
\end{aligned}
$$

and analogously

$$
\begin{aligned}
\hat{D}_{a b}^{\dagger} & =\sum_{k, m=0}^{N-1}|k\rangle_{a}\langle k|\otimes|(m-k) \bmod N\rangle_{b}\langle m| \\
& \equiv \sum_{k=0}^{N-1}|k\rangle_{a}\langle k| \otimes \hat{R}_{x}^{(b)}(-k),
\end{aligned}
$$

where the subscripts $a$ and $b$ indicate on which Hilbert space the given operator acts. Now we see that for $N>2$ the two operators $\hat{D}$ and $\hat{D}^{\dagger}$ do differ; they describe conditional shifts in opposite directions. We see that the generalization of the CNOT operator are the conditional shifts. The amount by which the target (in our case particle $b$ ) is shifted depends on the state of the control particle $(a)$ [for a pictorial representation of this gate see Fig. 1]

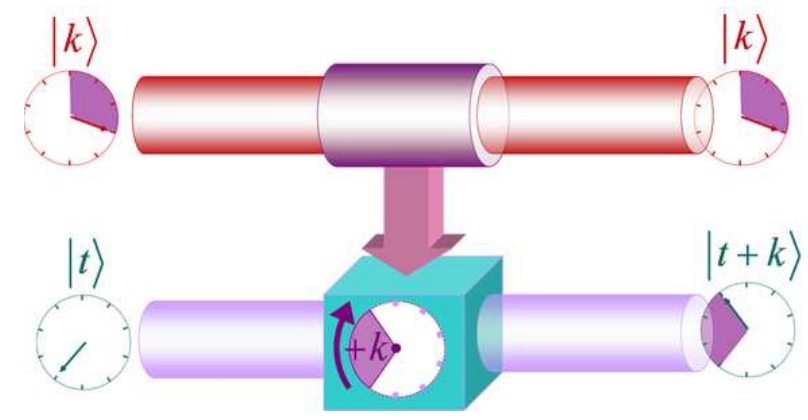

FIG. 1. Schematic description of the two-qudit conditional-shift gate.

\section{QUANTUM INFORMATION DISTRIBUTOR}

As shown in Ref. [15] quantum control over the quantum information can be achieved with the help of a quantum "machine", the so-called quantum information distributor (QID). The machine takes as an input a system qudit prepared in an unknown state $|\Psi\rangle_{1}$ and two ancilla qudits prepared in the state $|\Theta\rangle_{23}$ that play the role of quantum program (i.e., the CP map that has to be performed on the system qubit is encoded in this state). The action of the QID itself is described by a unitary operator $U_{123}$ acting on the Hilbert space that is a tensor product of the three qudits under consideration. This unitary operator can be expressed as a sequence of four controlled shifts $D_{k l}$, i.e.,

$$
\hat{U}_{123}=\hat{D}_{31} \hat{D}_{21}^{\dagger} \hat{D}_{13} \hat{D}_{12}
$$

The flow of information in the quantum distributor, as described by the unitary operator (3.1), is governed by the preparation of the distributor itself, i.e., by the choice of the program state $|\Theta\rangle_{23}$. In other words, we imagine the transformation (3.1) as a universal "processor" or distributor and the state $|\Theta\rangle_{23}$ as "program" through which the information flow is controlled.

We present the logical network for the QID in Fig. 2 The output state of the three-particle system after the four controlled shifts are applied is

$$
\left|\Omega^{(\text {out })}\right\rangle_{123}=\hat{D}_{31} \hat{D}_{21}^{\dagger} \hat{D}_{13} \hat{D}_{12}|\Psi\rangle_{1}|\Theta\rangle_{23}
$$

Note that the QID is covariant with respect to any choice of the state $|\Psi\rangle_{1}$ of data register (for more details see Ref. [15]).

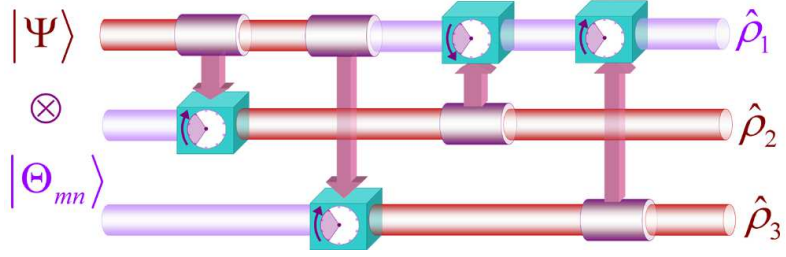

FIG. 2. Logical network for the quantum-information distributor. The network is composed of four conditional-shift gates. 


\section{A. Factorized program states}

Let us first assume that the two program qudits are in a pure state

$$
|\Theta\rangle_{23}=\left|x_{m}\right\rangle_{2}\left|p_{n}\right\rangle_{3}
$$

After the action of the QID the state $|\Omega\rangle_{123}=|\Psi\rangle_{1} \otimes$ $|\Theta\rangle_{23}$ transforms as

$$
\begin{aligned}
\left|\Omega^{(\text {out })}\right\rangle_{123} & =U_{123}|\Psi\rangle_{1}\left|x_{m}\right\rangle_{2}\left|p_{n}\right\rangle_{3} \\
& =\left[\hat{R}_{x}(m) \hat{R}_{p}^{\dagger}(n)|\Psi\rangle_{2}\right] \otimes\left|\Xi_{n m}\right\rangle_{31}
\end{aligned}
$$

So we can observe two actions of the QID on the input state: First, the state of the original qudit has been totally copied on the state of the second qudit. Simultaneously, the second qudit undergoes two rotations described by the operator $\hat{R}_{x}(m) \hat{R}_{p}^{\dagger}(n)$, where the values of the rotations are uniquely determined by the program state. Finally, the two remaining qudits (labeled as 1 and 3) became maximally entangled as the result of the action of the QID.

\section{B. Maximally entangled program states}

Let us assume that the QID state $|\Theta\rangle_{23}$ is initially prepared in the maximally entangled state $\left|\Xi_{m n}\right\rangle_{23}$ given by Eq. (2.9) Taking the original system to be prepared in the state $|\Psi\rangle_{1}$, i.e. the three qudits at the input are in the state

$$
|\Omega\rangle_{123}=|\Psi\rangle_{1} \otimes\left|\Xi_{m n}\right\rangle_{23}
$$

we find after the QID transformation the expression for the state vector of the three qudits

$$
\left|\Omega^{(\text {out })}\right\rangle_{123}=\left[\hat{R}_{x}^{\dagger}(n) \hat{R}_{p}^{\dagger}(m)|\Psi\rangle_{1}\right] \otimes\left|\Xi_{m n}\right\rangle_{23} .
$$

We see that if the program register is initially prepared in the maximally entangled state then the information encoded in the input state of the first (system) qudit will remain in this qudit, but the QID will induce a specific rotation on this qudit that is uniquely determined by the maximally entangled state of the program qudits. Interestingly enough, the program state is not changed at all in this case.

\section{Superposition of program states}

The complete set of maximally entangled states Eq. (2.9) is a basis for the two-qudit Hilbert space. Therefore, an arbitrary program state can be written in the form

$$
|\Theta\rangle_{23}=\sum_{m, n=0}^{N-1} d_{m n}\left|\Xi_{m n}\right\rangle
$$

and the corresponding evolution of the QID results in the state

$$
\begin{aligned}
\hat{U}|\Omega\rangle & =\sum_{m, n=0}^{N-1} d_{m n} \hat{R}_{x}^{\dagger}(n) \hat{R}_{p}^{\dagger}(m)|\Psi\rangle_{1} \otimes\left|\Xi_{m n}\right\rangle_{23} \\
& =\sum_{m, n=0}^{N-1} \tilde{d}_{m n}\left[\hat{R}_{p}^{\dagger}(n) \hat{R}_{x}(m)|\Psi\rangle_{2}\right] \otimes\left|\Xi_{n m}\right\rangle_{13}
\end{aligned}
$$

where $\tilde{d}_{m n}$ is the Fourier transformation of the coefficients $d_{m n}$ :

$\tilde{d}_{m n}=\frac{1}{N} \sum_{k, l=0}^{N-1} d_{k l} \exp \left[i \frac{2 \pi}{N}(k m+l n)\right]=F\left(d_{m n}\right)$

This last result is not surprising, since the complete set of factorized states $\left|x_{m}\right\rangle\left|p_{n}\right\rangle$ forms another orthonormal basis for the program space. If the program space is expanded in this basis Eq. (3.9) immediately results. What is interesting is that the program state that induces a specific operation on the first qudit performs an analogous (though not identical) operation on the second qudit. To see this we present the reduced density operators of these two qudits at the output of the QID [see Eqs. (3.8) and $(3.9)]$ :

$$
\begin{aligned}
& \hat{\rho}_{1}=\sum_{m, n=0}^{N-1}\left|d_{m n}\right|^{2} \hat{R}_{x}^{\dagger}(n) \hat{R}_{p}^{\dagger}(m)|\Psi\rangle\langle\Psi| \hat{R}_{p}(m) \hat{R}_{x}(n) ; \\
& \hat{\rho}_{2}=\sum_{m, n=0}^{N-1}\left|\tilde{d}_{m n}\right|^{2} \hat{R}_{x}(m) \hat{R}_{p}^{\dagger}(n)|\Psi\rangle\langle\Psi| \hat{R}_{p}(n) \hat{R}_{x}^{\dagger}(m) .
\end{aligned}
$$

We will use this property of the QID and it application as a measurement device that realizes a generalized measurement in our further analysis of the QID.

\section{The case of qubits}

We have seen that for qudits, there are two special bases that cause a set of operations to be performed on the input data state so that at the output, the transformed data state is disentangled from the output program state. In the case of qubits there is a third.

In this section let us change our notation to connect it to that usually employed for two-state systems. We shall denote the states $\left|x_{0}\right\rangle$ and $\left|x_{1}\right\rangle$ by $|0\rangle$ and $|1\rangle$, respectively. The states $\left|p_{0}\right\rangle$ and $\left|p_{1}\right\rangle$ can then be expressed as

$$
\begin{aligned}
& \left|p_{0}\right\rangle=\frac{1}{\sqrt{2}}(|0\rangle+|1\rangle) \\
& \left|p_{1}\right\rangle=\frac{1}{\sqrt{2}}(|0\rangle-|1\rangle) .
\end{aligned}
$$


The maximally entangled states $\left|\Xi_{m n}\right\rangle$ are just the Bell states. The actions of the product and maximally entangled program states can be expressed as

$$
\begin{aligned}
& U_{123}|\Psi\rangle_{1}\left|\Xi_{00}\right\rangle_{23}=|\Psi\rangle_{1}\left|\Xi_{00}\right\rangle_{23} \\
& U_{123}|\Psi\rangle_{1}\left|\Xi_{10}\right\rangle_{23}=\left(\sigma_{z}|\Psi\rangle_{1}\right)\left|\Xi_{10}\right\rangle_{23} \\
& U_{123}|\Psi\rangle_{1}\left|\Xi_{01}\right\rangle_{23}=\left(\sigma_{x}|\Psi\rangle_{1}\right)\left|\Xi_{01}\right\rangle_{23} \\
& U_{123}|\Psi\rangle_{1}\left|\Xi_{11}\right\rangle_{23}=-i\left(\sigma_{y}|\Psi\rangle_{1}\right)\left|\Xi_{11}\right\rangle_{23},
\end{aligned}
$$

and

$$
\begin{aligned}
U_{123}|\Psi\rangle_{1}|0\rangle_{2}\left|p_{0}\right\rangle_{3} & =|\Psi\rangle_{2}\left|\Xi_{00}\right\rangle_{13} \\
U_{123}|\Psi\rangle_{1}|0\rangle_{2}\left|p_{1}\right\rangle_{3} & =\left(\sigma_{z}|\Psi\rangle_{2}\right)\left|\Xi_{10}\right\rangle_{13} \\
U_{123}|\Psi\rangle_{1}|1\rangle_{2}\left|p_{0}\right\rangle_{3} & =\left(\sigma_{x}|\Psi\rangle_{2}\right)\left|\Xi_{01}\right\rangle_{13} \\
U_{123}|\Psi\rangle_{1}|1\rangle_{2}\left|p_{1}\right\rangle_{3} & =i\left(\sigma_{y}|\Psi\rangle_{2}\right)\left|\Xi_{11}\right\rangle_{13}
\end{aligned}
$$

There is now a second product basis that causes the transformed data state to emerge from output 3 . We have that

$$
\begin{aligned}
& U_{123}|\Psi\rangle_{1}\left|p_{0}\right\rangle_{2}|0\rangle_{3}=|\Psi\rangle_{3}\left|\Xi_{00}\right\rangle_{12} \\
& U_{123}|\Psi\rangle_{1}\left|p_{1}\right\rangle_{2}|0\rangle_{3}=\left(\sigma_{z}|\Psi\rangle_{3}\right)\left|\Xi_{10}\right\rangle_{12} \\
& U_{123}|\Psi\rangle_{1}\left|p_{0}\right\rangle_{2}|1\rangle_{3}=\left(\sigma_{x}|\Psi\rangle_{3}\right)\left|\Xi_{01}\right\rangle_{12} \\
& U_{123}|\Psi\rangle_{1}\left|p_{1}\right\rangle_{2}|1\rangle_{3}=i\left(\sigma_{y}|\Psi\rangle_{3}\right)\left|\Xi_{11}\right\rangle_{12}
\end{aligned}
$$

The additional basis suggests that it would be useful to examine program states that are superpositions of three states, one from each basis. Perhaps the simplest of these is the one that is a superposition of the states corresponding to the identity operator

$$
|\Theta\rangle_{23}=\alpha\left|\Xi_{00}\right\rangle_{23}+\beta|0\rangle_{2}\left|p_{0}\right\rangle_{3}+\gamma\left|p_{0}\right\rangle_{2}|0\rangle_{3},
$$

where $\left|\Xi_{00}\right\rangle_{a b}=(|00\rangle+|11\rangle) / \sqrt{2}$. The normalization condition for this state is

$$
|\alpha+\beta|^{2}+|\alpha+\gamma|^{2}+|\beta+\gamma|^{2}=2 .
$$

We hope that this state will lead to an output that consists of three approximate copies of the input data state, $|\Psi\rangle_{1}$, however, we find that this is not what happens. The reduced density matrix of the first output is

$$
\begin{aligned}
\hat{\rho}_{1} & =\left[\left|\alpha+\frac{(\beta+\gamma)}{2}\right|^{2}-\frac{|\beta+\gamma|^{2}}{4}\right] \hat{\rho}+\frac{|\beta+\gamma|^{2}}{2} \hat{\mathbb{1}} \\
& -\frac{\left(\beta \gamma^{*}+\beta^{*} \gamma\right)}{2} \sigma_{y} \hat{\rho} \sigma_{y},
\end{aligned}
$$

where we used the notation $\hat{\rho}=|\Psi\rangle\langle\Psi|$. Similar results are obtained for the reduced density matrices of outputs 2 and 3. As can be seen, while the first two terms are, in fact, an approximate copy of the input state, this is disturbed by the last term. Note that if either $\beta$ or $\gamma$ is zero, this term disappears and the device behaves as an approximate cloner.
A somewhat more successful example is given by a program state consisting of states each of which corresponds to a different operation, e.g.

$$
|\Theta\rangle_{23}=\alpha\left|\Xi_{00}\right\rangle_{23}+\beta|0\rangle_{2}\left|p_{1}\right\rangle_{3}+\gamma\left|p_{0}\right\rangle_{2}|1\rangle_{3},
$$

with the normalization condition

$$
|\alpha+\beta|^{2}+|\alpha+\gamma|^{2}+|\beta-\gamma|^{2}=2 .
$$

The first state in the superposition produces $|\Psi\rangle$ in output 1, the second $\sigma_{z}|\Psi\rangle$ in output 2, and the third $\sigma_{x}|\Psi\rangle$ in output 3. The single-qubit reduced density matrices resulting from this program state are

$$
\begin{aligned}
& \hat{\rho}_{1}=\left[\left|\alpha+\frac{(\beta+\gamma)}{2}\right|^{2}-\frac{|\beta-\gamma|^{2}}{4}\right] \hat{\rho}+\frac{|\beta-\gamma|^{2}}{2} \hat{\mathbb{1}} ; \quad(3.22) \\
& \hat{\rho}_{2}=\left[\left|\beta+\frac{(\alpha-\gamma)}{2}\right|^{2}-\frac{|\alpha+\gamma|^{2}}{4}\right] \sigma_{z} \hat{\rho} \sigma_{z}+\frac{|\alpha+\gamma|^{2}}{2} \hat{\mathbb{1}} ; \\
& \hat{\rho}_{3}=\left[\left|\gamma+\frac{(\alpha-\beta)}{2}\right|^{2}-\frac{|\alpha+\beta|^{2}}{4}\right] \sigma_{x} \hat{\rho} \sigma_{x}+\frac{|\alpha+\beta|^{2}}{2} \hat{\mathbb{1}} .
\end{aligned}
$$

In this case we do obtain an approximate version of $|\Psi\rangle$ in output 1, an approximate version of $\sigma_{z}|\Psi\rangle$ in output 2, and an approximate version of $\sigma_{x}|\Psi\rangle$ in output 3. A simple way to see how the accuracy of the approximations in the different outputs is constrained, is to define the fidelities

$$
\begin{aligned}
& F_{1}=\left\langle\Psi\left|\hat{\rho}_{1}\right| \Psi\right\rangle ; \\
& F_{2}=\left\langle\sigma_{z} \Psi\left|\hat{\rho}_{2}\right| \sigma_{z} \Psi\right\rangle ; \\
& F_{3}=\left\langle\sigma_{x} \Psi\left|\hat{\rho}_{3}\right| \sigma_{x} \Psi\right\rangle,
\end{aligned}
$$

and to note that $F_{1}+F_{2}+F_{3}=2$. Each of the fidelities lies between $1 / 2$ and 1 . Noting that a completely noisy output of $\hat{\mathbb{1}} / 2$, containing no information about the input, corresponds to a fidelity of $1 / 2$, we see that if one of the fidelities is 1 , containing perfect information about the input, the others are $1 / 2$, and contain no information. Thus, we have a kind of conservation of information, the more accurate one output becomes, the less accurate the others become in order to compensate. If the fidelities are equal, then each is equal to $2 / 3$. This is the fidelity of state estimation, and hence cloning, that would be achieved by simply measuring the input qubit.

\section{QUANTUM PROPENSITIES}

According to Wódkiewicz [28], propensity means the tendency (or probability) of a measured object to take up certain states prescribed by a measuring device. Let the measuring device - the so called quantum ruler - be in a pure state $|\Phi\rangle$. The quantum-ruler state can be "shifted" 
by an action of some generalized displacement operator $\hat{D}(g)$, where $g$ is an element of a group $G$. If the measured system is in a pure state $|\Psi\rangle$, then its probability to be in the ruler state shifted by $g$ (i.e., the propensity) is

$$
P_{\Phi, \Psi}(g)=|\langle\Psi|\hat{D}(g)| \Phi\rangle|^{2},
$$

whereas if the system is in a mixed state described by the density operator $\hat{\rho}$, the propensity is

$$
P_{\Phi, \rho}(g)=\operatorname{Tr}\left(\hat{\rho} \hat{D}(g)|\Phi\rangle\langle\Phi| \hat{D}^{+}(g)\right)
$$

In our case, that of a finite dimensional Hilbert space, the group $G$ will be formed by discrete translations on a torus: if $g_{1} \equiv\left(n_{1}, m_{1}\right)$ and $g_{2} \equiv\left(n_{2}, m_{2}\right)$ are elements of $G$, then their group product is $g_{1} g_{2} \equiv$ $\left(\left(n_{1}+n_{2}\right) \bmod N,\left(m_{1}+m_{2}\right) \bmod N\right)$. The corresponding displacement operator is then given by the expression $\hat{R}_{x}(n) \hat{R}_{p}(m)$. We see that while the displacement is not a representation of the group $G$ in the Hilbert space under consideration, nevertheless it is representation of this group in a ray space, which enables us to define the propensity uniquely. For a pure state $|\Psi\rangle$ we can write the propensity in the form (see Ref. [24]):

$$
P_{\Phi, \Psi}(n, m)=\left|\left\langle\Psi\left|\hat{R}_{x}(n) \hat{R}_{p}(m)\right| \Phi\right\rangle\right|^{2} .
$$

In the case of a statistical mixture described by the density operator $\hat{\rho}$ the corresponding propensity reads

$$
P_{\Phi, \rho}(n, m)=\operatorname{Tr}\left(\hat{\rho} \hat{R}_{x}(n) \hat{R}_{p}(m)|\Phi\rangle\langle\Phi| \hat{R}_{p}^{\dagger}(n) \hat{R}_{x}^{\dagger}(m)\right)
$$

\section{A. Propensities and POVM measurements}

The propensities as defined above are in fact results of so-called generalized (positive operator value measure POVM) measurements (e.g., see Ref. [29]). To see this let us recall that

$$
\hat{F}_{m n}=\hat{R}_{x}(n) \hat{R}_{p}(m)|\Phi\rangle\langle\Phi| \hat{R}_{p}^{\dagger}(m) \hat{R}_{x}^{\dagger}(n)
$$

where $|\Phi\rangle$ is a ruler state are positive operators and they fulfill the condition

$$
\sum_{m n} \hat{F}_{m n}=N \hat{\mathbb{1}}
$$

So the operators $\hat{F}_{m n}$ (or more specifically the operators $\left.\hat{f}_{m n}=\hat{F}_{m n} / N\right)$ form a complete set that can be used for a complete measurement of the state of a qudit. We note that other operators of the form (4.5), e.g.,

$$
\hat{F}_{m n}=\hat{R}_{x}(m) \hat{R}_{p}^{\dagger}(n) \hat{\rho} \hat{R}_{p}(n) \hat{R}_{x}^{\dagger}(m)
$$

also realize a POVM measurement.

\section{B. $Q$ function in discrete phase space}

In an analogy with a continuous $(q, p)$ phase space, where the $Q$ function (Husimi function) is defined as the propensity of a state to be in the vacuum state, we define the discrete $Q$ function as propensity (4.1)

$$
Q(n, m) \equiv P_{\Phi, \rho}(n, m)
$$

with the quantum ruler being in a "vacuum" state. The problem is how to define a vacuum state corresponding to a finite-dimensional Hilbert space.

Before specifying the ruler state, we will mention several properties of discrete $Q$ functions. If we assume that the ruler state $|\Phi\rangle$ is chosen (i.e., the vacuum state is specified) then the $Q$ function has the following properties:

(i) it is uniquely defined;

(ii) it is non-negative;

(iii) it is normalized to $N$

$$
\sum_{n, m} Q(n, m)=N
$$

(iv) for properly chosen ruler states $|\Phi\rangle$ the information about a system state can be completely reconstructed from the corresponding $Q$ function.

\section{Ruler state}

In analogy with the continuous limit, where the ruler state associated with a Husimi function is the ground (vacuum) state of the harmonic oscillator, let us consider following requirements on the ruler state: (i) it should be in some sense centered at origin of phase space [i.e., the point $(0,0)]$, (ii) it should be "symmetric" with regards to the quantities $X$ and $P$, i.e., its wave function should have similar form in both representations (perhaps up to scalings), and (iii) it should be in some sense a minimum uncertainty state, which means that in the phase space it should be represented by a peak which is as narrow as possible. As shown in Ref. [19] all the above properties are fulfilled by the ground state of the Hamiltonian

$$
\hat{H}_{0}=-\cos \left(\frac{2 \pi}{N} \hat{X}\right)-\cos \left(\frac{2 \pi}{N} \hat{P}\right) .
$$

We will use this ground state as the ruler state in our forthcoming considerations. 


\section{POVM MEASUREMENT VIA QID}

Let us now study the action of the quantum information distributor when the two ancillary qudits are prepared in a superposition state

$$
|\Theta\rangle_{23}=\left(\alpha\left|\Xi_{00}\right\rangle_{23}+\beta\left|x_{m}\right\rangle_{2}\left|p_{n}\right\rangle_{3}\right)
$$

with the two real amplitudes $\alpha$ and $\beta$ satisfying the normalization condition

$$
\alpha^{2}+\beta^{2}+\frac{2 \alpha \beta}{N} \cos \left(\frac{2 \pi}{N} n m\right)=1 .
$$

With this program state the QID acts on the input data qudit $|\Psi\rangle_{1}=\sum_{k} c_{k}\left|x_{k}\right\rangle$ so that at the output the three qudits are in the following states:

$$
\begin{aligned}
\hat{\rho}_{1}= & \left(1-\beta^{2}\right) \hat{\rho}+\frac{\beta^{2}}{N} \hat{\mathbb{1}} ; \\
\hat{\rho}_{2}= & \left(1-\alpha^{2}\right) \hat{R}_{x}(m) \hat{R}_{p}^{\dagger}(n) \hat{\rho} \hat{R}_{p}(n) \hat{R}_{x}^{\dagger}(m)+\frac{\alpha^{2}}{N} \hat{\mathbb{1}} ; \\
\hat{\rho}_{3}= & \left(1-\alpha^{2}-\beta^{2}\right) \hat{R}_{x}(m) \hat{R}_{p}(n) \hat{\rho}^{\mathrm{T}} \hat{R}_{p}^{\dagger}(n) \hat{R}_{x}^{\dagger}(m) \\
& +\frac{\alpha^{2}+\beta^{2}}{N} \hat{\mathbb{1}},
\end{aligned}
$$

where $\hat{\rho}=|\Psi\rangle\langle\Psi|$ and $\hat{\rho}^{\mathrm{T}}$ is the transpose of the density operator $\hat{\rho}=\sum_{k, k^{\prime}} c_{k} c_{k^{\prime}}^{*}\left|x_{k}\right\rangle\left\langle x_{k^{\prime}}\right|$. That is, in the basis $\left|x_{k}\right\rangle$ the transposed density operator reads $\hat{\rho}^{\mathrm{T}}=$ $\sum_{k, k^{\prime}} c_{k}^{*} c_{k^{\prime}}\left|x_{k}\right\rangle\left\langle x_{k^{\prime}}\right|$.
The action of the QID discussed earlier, allows us to reconstruct partially the state of the measured system without a total "destruction" of the state of the data register. Specifically, from Eq. (5.3) it follows that the entangled component of the program register (represented by the state $\left|\Xi_{00}\right\rangle_{23}$ ) dictates how "much" of the original information encoded in the qudit 1 is transferred from the data register to the program register at the output of the QID. For instance, if the amplitude $\alpha$ is equal to unity (i.e., $\beta=0$ ) then the data register is not perturbed at all, and no information is transferred. On the other hand, for $\alpha<1$ some of the information from the data is transferred to the program at the expense of noise introduced into the data register. The trade-off between the information transfer and the noise introduced into the data register is nicely seen from Eq. (5.3). The amount of noise that is transferred into the first (data) qudit is dictated by the amplitude $\beta$ that weights the factorizable contribution to the program state, i.e. $\left|x_{n}\right\rangle_{2}\left|p_{m}\right\rangle_{3}$. Moreover this specific state also determines operations (rotations) that are performed on program qudits.

In order to illustrate the action of the QID we plot in Fig. $3 Q$ functions of an input qudit, that is, initially prepared in the ground state of Hamiltonian (4.10), as well as the three output qudits. The ruler state is chosen to be again the ground state of Hamiltonian (4.10). The Husimi functions do correspond to the situation when a POVM measurement is performed on the density operator $\hat{\rho}_{j}(j=1,2,3)$ given by Eqs. (5.3) - (5.5), respectively.

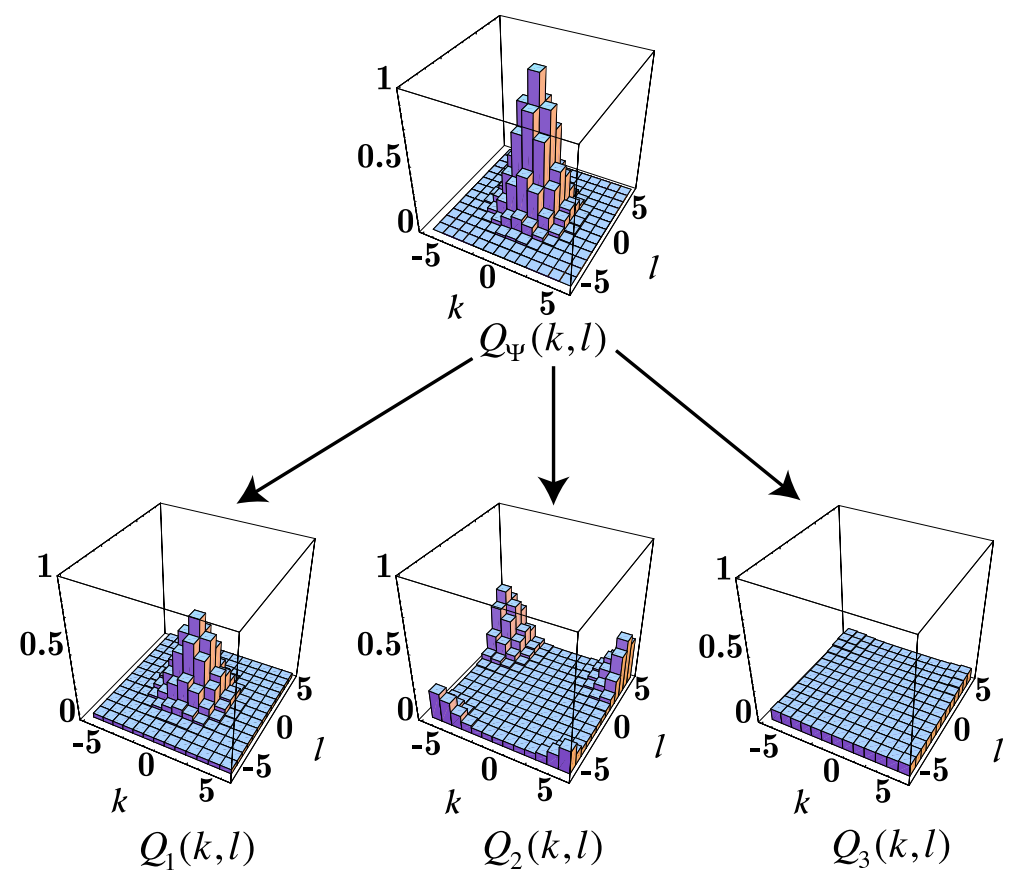


FIG. 3. Husimi functions of the input state of the data qudit and the output qudits. The input data qudit is initially prepared in the ground state of the Hamiltonian (4.10) while the auxiliary system (ancilla) is initially prepared in the state $|\Theta\rangle=0.75\left|\Xi_{00}\right\rangle-0.64\left|x_{7}\right\rangle\left|p_{5}\right\rangle$. The top graph, labeled $Q_{\Psi}(k, l)$, represents the Husimi function of the initial state of the data qudit. The three graphs, labeled $Q_{1}(k, l), Q_{2}(k, l)$ and $Q_{3}(k, l)$, represent the Husimi functions of reduced states $\hat{\rho}_{1}, \hat{\rho}_{2}$ and $\hat{\rho}_{3}$ of the composite system that are given by Eqs. (5.3)-(5.5), respectively.

\section{EFFECT OF MEASUREMENT}

It is obvious from expression (5.4) that if the von Neumann measurement using the projector $|\Phi\rangle_{2}\langle\Phi|$ (i.e., projecting on the ruler state) on qudit 2 is performed then this measurement results in a reconstruction of the Husimi function of the original data state affected by the amount of noise determined by the particular value of $\alpha$. In other words, this projective measurement will result in the reconstruction of the Husimi function of the operator $\hat{\rho}_{2}^{\text {(out) }}=\left(1-\alpha^{2}\right) \hat{\rho}+\frac{\alpha^{2}}{N} \hat{\mathbb{1}}$. Certainly, the state of the data register is then affected not only by the action of the QID but also by the effect of the projective measurement performed on the second qudit.

To understand the role of the projective measurement performed on the program register on the state of the data register at the output of the QID, let us consider the following. We will study the action of the quantuminformation distributor when the two ancillary qudits are prepared in a superposition state given by Eq. (5.1) With this program state the QID acts on the input data qudit $|\Psi\rangle_{1}=\sum_{k} c_{k}\left|x_{k}\right\rangle$ so that at the output the three qudits are in the state:

$$
\begin{aligned}
\left|\Omega^{(\text {out })}\right\rangle_{123} & =U_{123}|\Psi\rangle_{1}\left[\alpha\left|\Xi_{00}\right\rangle_{23}+\beta\left|x_{m}\right\rangle_{2}\left|p_{n}\right\rangle_{3}\right] \\
& =\alpha|\Psi\rangle_{1}\left|\Xi_{00}\right\rangle_{23}+\beta\left[\hat{R}_{x}(m) \hat{R}_{p}^{\dagger}(n)|\Psi\rangle\right]_{2}\left|\Xi_{n m}\right\rangle_{31}
\end{aligned}
$$

Then we will assume that both program qudits are measured projectively. Qudit 2 is projected in the ruler state $|\Phi\rangle_{2}=\sum_{k} f_{k}\left|x_{k}\right\rangle_{2}$ while the qudit 3 is projected on the transposed ruler state $\left|\Phi^{\mathrm{T}}\right\rangle_{3}:=\sum_{k} f_{k}^{*}\left|x_{k}\right\rangle_{3}$. Schematically this situation is depicted in Fig. 4

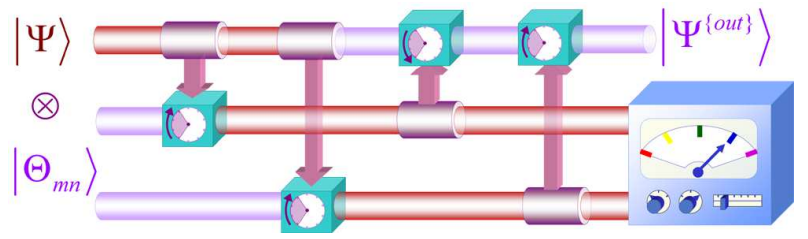

FIG. 4. Logical network for the quantum information distributor with a projective measurement performed on the program register.

The data qudit after the action of the QID and this projective measurement reads

$$
\begin{aligned}
& \left|\Psi^{(\text {out })}\right\rangle_{1} \simeq_{2}\left\langle\left.\Phi\right|_{3}\left\langle\Phi^{\mathrm{T}} \mid \Omega^{(\text {out })}\right\rangle_{123}\right. \\
& =\frac{\alpha}{\sqrt{N}}|\Psi\rangle_{1}+\frac{\beta\left\langle\Phi\left|\hat{R}_{x}(m) \hat{R}_{p}^{\dagger}(n)\right| \Psi\right\rangle}{\sqrt{N}} \hat{R}_{x}^{\dagger}(m) \hat{R}_{p}(n)|\Phi\rangle_{1} .
\end{aligned}
$$

This means that by acquiring knowledge of a particular value of the Husimi functions of the second and the third qudits, the data qudit "collapses" into the state (6.2). The disturbance of the original data state depends on the value of $\alpha$, the particular point $(m, n)$ at which the Husimi functions of the program qudits are measured and the specific choice of the ruler state.

\section{CONCLUSION}

Here we have shown how a simple quantum device, the quantum-information distributor, can both distribute and process quantum-information. This device was discussed in Ref. [15], and it was shown there that the flow of information was controlled by a program state. In this paper, we have considered a much wider class of program states. Besides moving the quantum information between outputs, they allow us to, in addition, apply shift operators to the input data. This, in turn, makes it possible to use the QID to measure the discrete $Q$ function of the input data, which is equivalent to realizing a class of POVM operators. Another possibility, is to split the input into two parts, find the $Q$ function of one part and retain the other part. There is a tradeoff involved: the more information that is retained, the more smeared is the $Q$ function, and the better the $Q$ function, the more distorted is the information in the retained qudit. Thus, the QID provides us with a very flexible programmable quantum-information processing device, which has a number of useful applications.

\section{ACKNOWLEDGMENTS}

This work was supported in part by the European Union project QGATES and by the grant agency VEGA of the Slovak Academy of Sciences and by NSF grant No. PHY-0139692. V.B. would like to thank the Science Foundation Ireland for support.

[1] H. K. Cummins, C. Jones, A. Furze, N. F. Soffe, M. Mosca, J. M. Peach, and J. A. Jones, Phys. Rev. Lett. 88, 187901 (2002). 
[2] A. Lamas-Linares, Ch. Simon, J.C. Howell, and D. Bouwmeester, Science 296, 712 (2002).

[3] F. De Martini, V. Bužek, F. Sciarrino, and C. Sias, Nature 419 No. 6909, 815 (2002).

[4] V. Bužek and M. Hillery, Phys. Rev. A 54, 1844 (1996).

[5] N. Gisin and S.Massar Phys. Rev. Lett. 79, 2153 (1997).

[6] R.F. Werner Phys. Rev. A 58, 1827 (1998).

[7] G. Harel and V.M. Akulin, Phys. Rev. Lett. 82 , 1 (1999).

[8] S. Lloyd and S.L. Braunstein, Phys. Rev. Lett. 82 , 1784 (1999).

[9] B. Hladký, G. Drobný, and V. Bužek Phys. Rev. A 61, 022102 (2000).

[10] M. A. Nielsen and I. L. Chuang, Phys. Rev. Lett. 79, 321 (1997).

[11] G. Vidal, L. Masanes, and J.I. Cirac, Phys.Rev.Lett. 88 047905 (2002)

[12] M. Hillery, V. Bužek, and M. Ziman, Forstschritte der Physik 49, 987-992 (2001)

[13] M. Hillery, V. Bužek, and M. Ziman, Phys.Rev A 65, $022301(2002)$

[14] M. Hillery, M. Ziman, and V.Bužek, Phys. Rev. A 66, 042302 (2002)

[15] S.L. Braunstein, V. Bužek and M. Hillery, Phys. Rev. A 63, 052313 (2001).

[16] V. Bužek, A. D. Wilson-Gordon, P. L. Knight and W. K. Lai, Phys. Rev. A 45, 8079 (1992).
[17] D. T. Pegg and S. M. Barnett, Europhys. Lett. 6, 483 (1988); Phys. Rev. A 39, 1665 (1989); D. T. Pegg, J. A. Vaccaro and S. M. Barnett, J. Mod. Opt. 37, 1703 (1990).

[18] W. K. Wootters, Ann. Phys. 176, (NY), 1 (1987).

[19] T. Opatrný, V. Bužek, J. Bajer, and G. Drobný, Phys. Rev. A 52, 2419 (1995)

[20] U. Leonhardt, Phys. Rev. Lett. 74, 4101 (1995).

[21] M. Koniorczyk, V. Bužek, and J. Janszky, Phys. Rev. A 64, 034301 (2001).

[22] J. P. Paz Phys. Rev. A 65, 062311 (2002).

[23] D. Galetti and A. F. R. de Toledo Piza, Physica 149A, 267 (1988).

[24] V. Bužek, C. H. Keitel and P. L. Knight, Phys. Rev. A 51, 2575 (1995).

[25] G. Alber, A. Delgado, N. Gisin, and I. Jex, J. Phys. A 34, 8821 (2001).

[26] V. Vedral, A. Barenco, and A. Ekert, Phys. Rev. A 54, 147 (1996).

[27] A. D. Pittenger, An Introduction to Quantum Computing (Birkhäuser, Boston, 2000).

[28] K. Wódkiewicz, Phys. Rev. Lett. 52, 1064 (1984); Phys. Lett. A 115, 304 (1986); Phys. Lett. A 129, 1 (1988).

[29] See for example: M.A. Nielsen and I.L. Chuang, Quantum Computation and Quantum Information (Cambridge University Press, Cambridge, 2000). 